\title{
Effects of Quadrat Size and Shape, Initial Epidemic Conditions, and Spore Dispersal Gradient on Spatial Statistics of Plant Disease Epidemics
}

\author{
X.-M. Xu and M. S. Ridout
}

Horticulture Research International, East Malling, West Malling, Kent, ME19 6BJ, United Kingdom. Accepted for publication 22 March 2000.

\begin{abstract}
Xu, X.-M., and Ridout, M. S. 2000. Effects of quadrat size and shape, initial epidemic conditions, and spore dispersal gradient on spatial statistics of plant disease epidemics. Phytopathology 90:738-750.

The spatiotemporal spread of plant diseases was simulated using a stochastic model to study the effects of initial conditions (number of plants initially infected and their spatial pattern), spore dispersal gradient, and size and shape of sampling quadrats on statistics describing the spatiotemporal dynamics of epidemics. The spatial spread of disease was simulated using a half-Cauchy distribution with median dispersal distance $\mu$ (units of distance). A total of 54 different quadrat types, including 23 distinct sizes ranging from 4 to 144 plants, were used to sample the simulated epidemics. A symmetric form of the binary power law with two parameters $(\alpha, \beta)$ was fitted to the sampled epidemic data using each of the 54 quadrats for each replicate simulation run. The $\alpha$ and $\beta$ estimates were highly correlated positively with each other, and their estimates were comparable to those estimated from observed epidemics. Intraclass correlation $(\kappa)$ was calculated for each quadrat
\end{abstract}

ABSTRACT type; $\kappa$ decreased exponentially with increasing quadrat size. An asymmetric form of the binary power law with three parameters $\left(\alpha_{1}, \beta_{1}\right.$, $\beta_{2}$ ) was used to relate $\kappa$ to the disease incidence $(\mathrm{p}) ; \beta_{1}$ was highly correlated to $\beta$ : $\beta_{1} \approx \beta-1$. In general, initial conditions and quadrat size affected $\alpha, \beta, \alpha_{1}, \beta_{1}$, and $\beta_{2}$ greatly. The parameter estimates increased as quadrat size increased, and the relationships were described well by a linear regression model on the logarithm of quadrat size with the slope or intercept parameters dependent on initial conditions and $\mu$. Compared with initial conditions and quadrat size, the overall effects of $\mu$ and quadrat shape were generally small, although within each quadrat size and initial condition they could be substantial. Quadrat shape had the greatest effect when the quadrat was long and thin. The relationship of the index of dispersion $(D)$ to $p$ and quadrat size was determined from the $\alpha$ and $\beta$ estimates. $D$ was greatest when $p$ was 0.5 and decreased when $p$ approached 0 or 1 . It increased with quadrat size and the rate of the increase was maximum when $p$ was 0.5 and decreased when $p$ approached 0 or 1 .
Characterization of the spatiotemporal dynamics of plant disease incidence and severity over time and space is of fundamental importance in epidemiology for describing and under-standing the development of diseases, for evaluating treatments $(12,16,37)$, and for developing efficient sampling schemes $(2,10,18)$. Many statistical methods have been developed and used to analyze the spatiotemporal dynamics of plant disease epidemics and have been reviewed recently $(11,16,25,37)$.

The beta-binomial distribution is more appropriate than the negative binomial distribution for examining spatial patterns of disease incidence for the presence of aggregation $(9,16)$. An alternative measure of variability is the intraclass correlation (4), which measures the correlation between the binary responses (diseased or not diseased) of individual plants within a sampling unit. The dispersion or aggregation parameter of the beta-binomial distribution is related mathematically to the intraclass correlation coefficient. Spatial autocorrelation is used to determine the spatial dependence of disease incidence over various spatial lags $(1,5,17)$. The binary form of the power law relationship, modified from Taylor's power law (29), has been used to investigate the temporal dynamics of aggregation of diseased plants $(8,16)$.

These spatiotemporal statistics and models are very useful in describing and summarizing observed epidemics. They can also

Corresponding author: X.-M. Xu; E-mail address: xangming.xu@hri.ac.uk

Publication no. P-2000-0502-02R

(C) 2000 The American Phytopathological Society be used in comparative epidemiology to characterize individual epidemics, to identify similarities and differences between epidemics, and, thus, to identify the key determinants of epidemic development $(13,14)$. Their usefulness in comparative epidemiology, however, depends critically on our understanding of their relationships with the underlying biological and physical processes and the relative effects of biological and physical processes on these statistics. Using an individual-based and spatially explicit stochastic simulation model, Xu and Ridout (33) investigated the effects of initial epidemic conditions (initial number of infected plants and their spatial pattern), sporulation rate, and spore dispersal gradient on the spatiotemporal dynamics of plant disease epidemics. They demonstrated clearly the importance of initial epidemic conditions and spore dispersal gradient in influencing intraclass correlation (based on a $5 \times 5$ quadrat) and spatial autocorrelation, agreeing with Yang (35).

The objective of this paper was to study the effects of sampling unit size and shape on intraclass correlation and the binary form of the power law relationship under various initial epidemic conditions using the simulation model described previously (33). The simulation model is not intended to be an exact model of any specific epidemic, but it reflects the main processes involved in the development of an epidemic. Intraclass correlation was calculated from the simulated epidemic data; the binary form of the power law was fitted to the simulated data. The relationships of the calculated intraclass correlation and the estimated binary power law parameters to the underlying biological and physical processes and to quadrat size and shape were investigated. 


\section{THEORY AND APPROACHES}

The binary form of the power law relationship. Taylor's power law (29) has been extended to relate the observed variance $\left(V_{0}\right)$ of disease incidence between quadrats to the variance expected $\left(V_{R}\right)$ if a binomial distribution is assumed for the number of diseased plants per quadrat $(8,16)$. This relationship is called the binary form of the power law and can be used to investigate the temporal dynamics of aggregation of diseased plants. There are two forms of this relationship. One is a symmetric form:

$$
V_{0}=A V_{R}^{\beta}=A[p(1-p) / n]^{\beta}
$$

in which $p$ is the disease incidence and $n$ is the total number of plants per quadrat. The other is a more general (potentially) asymmetric form:

$$
V_{0}=A p^{\beta_{1}}(1-p)^{\beta_{2}}
$$

in which quadrat size $(n)$ is not included explicitly but is incorporated into $A$.

The following discussion uses equation 1 only. When $V_{0}$ is greater than $V_{R}$, aggregation is indicated. Thus the condition for aggregation is

$$
V_{0} / V_{R}=A[p(1-p) / n]^{\beta-1}>1
$$

This variance/variance ratio is called the index of dispersion $(D)$ $(3,16)$ and provides a measure of the degree of aggregation of diseased plants. The value of $D$ is determined jointly by $A, n$ and $\beta$; it is not possible to infer $D$ from either $A$ or $\beta$ alone.

If $D$ is plotted against $p$, parameter $A$ affects only the amplitude of the curve, whereas $\beta$ determines its shape. The greater the value of $A$, the greater the value of $D$. The greater the value of $\beta$, the lower the value of $D$, since $p(1-p) / n<1$. If $A<1, \beta$ must be less than 1 to satisfy equation 3 . When $\beta=1, D$ is constant and equal to $A$; when $A$ and $\beta=1$, there is no aggregation or overdispersion $(D=1)$. When $\beta>1, D$ increases with $p$, reaches a maximum, $A[1 /(4 \mathrm{n})]^{\beta-1}$, at $p=0.5$, and then decreases toward 0 as $p$ approaches 1 . However, for $\beta<1, D$ increases as $p$ approaches 0 or 1 . For all the plant disease incidence data that have been fitted to the binary form of the power law so far, estimates of $\beta$ have been greater than or equal to $1(16,17,19)$.

Another related statistic based on quadrat sampling is intraclass correlation $(\kappa)$, which measures the tendency of the plants within a sampling quadrat to have a similar disease status. Positive values of $\kappa$ indicate aggregation of disease; $\kappa$ is related to the binary form of the power law and index of dispersion (16):

$$
\kappa=(D-1) /(n-1)
$$

where $D$ is the index of dispersion and $n$ is the quadrat size.

The simulation model and details of simulation runs. Details of the model have been described previously (33). The model is a two-dimensional stochastic spatial contact model (21) in which the distance that spores are dispersed follows a specific longtailed probability distribution, the half-Cauchy distribution (26).

The spread of disease is modeled on a rectangular lattice of plants consisting of $n_{r}=150$ rows with $n_{c}=150$ plants in each row. The distance between adjacent plants within a row is $d_{\mathrm{c}}=1$, and the distance between rows is $d_{r}=1$. Each plant is assumed to consist of $n_{S}=30$ units that are susceptible to the pathogen. The epidemic is initiated by a single infected unit on one or more plants. These initially infected plants may be located systematically or randomly within the lattice.

Having completed a latent period of $t_{L}=5$ days, an infected unit becomes infectious for $t_{I}=10$ days, producing viable spores according to a Poisson process at a rate of $\lambda$ (per day, sporulation rate). Spores are assumed to be dispersed independently of one another and to travel in straight lines of random orientation, with the dispersal distance varying randomly according to a halfCauchy distribution (26), with median dispersal distance parameter $\mu$. If the spore falls beyond the end of a row by a distance of more than $d_{c} / 2$, or beyond the extreme rows by a distance of more than $d_{r} / 2$, it is ignored. Otherwise it lands on a randomly selected unit on the nearest plant and this unit becomes infected if it has not been infected previously; otherwise the spore has no effect. The model allows for the spread of disease within as well as between plants. Random numbers were generated using the uniform random number generator of Wichmann and Hill (32).

The simulation variables included the initial number of infected plants $\left(Y_{0}\right)$ and their spatial pattern $\left(Y_{S}\right)$ and the median spore dispersal parameter $(\mu)$. In the present study, the sporulation rate was constant $\left(\lambda=0.7\right.$ day $\left.^{-1}\right)$, as previous studies (33) showed that $\lambda$ did not affect spatial statistics describing the dynamics of epidemics directly, only indirectly via disease incidence. The initial number of infected plants $\left(Y_{0}\right)$ was 1,4 , or 9 , and each had a single infected unit when it entered its infectious period. The initial infected plants were located with one of three spatial patterns $\left(Y_{S}\right)$ : clumped, regular, and random. With $Y_{0}=1$, the regular and clumped patterns were the same, i.e., the infected plant was placed in the (approximate) center of the grid. With $Y_{0}=$ 4 or 9 , the infected plants were placed in the (approximate) central $2 \times 2$ or $3 \times 3$ grid for the clumped pattern. For the regular pattern, the central $120 \times 120$ grid was partitioned into 4 or 9 equal-sized square blocks and one infected plant was placed at the (approximate) center of each block. For the random pattern, locations for the infected plants were chosen within the central $120 \times 120$ grid of plants using a uniform random number generator (32) for each simulation run. Thus, in total, there were eight combinations of $Y_{0}$ and $Y_{S}$, i.e., eight initial epidemic conditions $(Y)$. Five values of $\mu$ were used: $0.25,0.50,1.0,2.0$, and 4.0 , in the same units as $d_{r}$. Thus, there were in total $40(8 \times$ 5) combinations of the three simulation variables $\left(Y_{0}, Y_{S}\right.$, and $\left.\mu\right)$. For each combination of $Y_{0}, Y_{\mathrm{S}}$, and $\mu$, the model was simulated 50 times, with each run terminating at various times, ranging from 48 to 145 days, to ensure that the final disease incidence was greater than $80 \%$. This ensured a wide range of disease incidence values for estimating the intraclass correlation and variance-mean relationship.

Statistical analysis. The effects of the initial number of infected plants $\left(Y_{0}\right)$ and their spatial configuration $\left(Y_{S}\right)$, the dispersal parameter $(\mu)$, and the sampling quadrat size $\left(Q_{S}\right)$ and shape $\left(Q_{P}\right)$ on intraclass correlation and on the binary power law relationship describing the spatiotemporal dynamics of the simulated epidemics were studied.

Temporal rates of disease progress were not calculated because previous studies (33) showed that the rate of increase in disease incidence $(p)$ over time was well described by the logistic model, with the rate determined mainly by $\lambda$ and $\mu$. The binary form of the power law was fitted to the data sampled from each simulated epidemic using quadrats of various sizes and shapes. Plants with at least one unit infected were classified as diseased. Although the epidemic was simulated on a $150 \times 150$ grid, disease incidence data were sampled only from the central $120 \times 120$ grid at an interval of 3 days, using a contiguous array of 1 of 54 quadrat types $(Q)$. The 54 quadrat types were chosen according to the following criteria: (i) the length and width of the quadrat were exact divisors of 120 , so that an array of contiguous quadrats covered the whole central grid; (ii) the maximum quadrat area was 144 plants; (iii) all possible square quadrats were included; and (iv) rectangular quadrats were included if there were at least two quadrat shapes of the same area.

At each sampling time, the variance $\left(\mathrm{V}_{0}\right)$ of disease incidence among quadrats and intraclass correlation, calculated as $\kappa=(\mathrm{D}-$ $1) /(\mathrm{n}-1)=\left(\mathrm{V}_{0} / \mathrm{V}_{R}-1\right) /(\mathrm{n}-1)$, were estimated for each of the 54 quadrat types; these calculations were implemented directly in the simulation program. Equations 1 and 2 were fitted to the data obtained from each simulated epidemic. However, there was little difference in the goodness-of-fit of the two models. Therefore, only the results from fitting equation 1 are presented in this paper. 
For parameter estimation, a linearized form of equation 1 was used:

$$
\ln \left(V_{0}\right)=\ln (A)+\beta \ln [p(1-p) / n]=\alpha+\beta \ln [p(1-p) / n]
$$

where $\alpha=\ln (A)$.

As in the previous paper (33), the asymmetric form of the binary power law (equation 2 ) was used to relate $\kappa$ to disease incidence $(p)$ :

$$
\kappa=A_{1} p^{\beta_{1}}(1-p)^{\beta_{2}}
$$

where $A_{1}, \beta_{1}$, and $\beta_{2}$ are parameters to be estimated. A linearized form of equation 6 was used for parameter estimation:

$$
\ln (\kappa)=\alpha_{1}+\beta_{1} \ln (p)+\beta_{2} \ln (1-p)
$$

where $\alpha_{1}=\ln \left(A_{1}\right)$. Equations 5 and 7 were fitted to the data for each simulated epidemic by least squares regression, assuming a random error with constant variance. In fitting the two models, data points with $p<0.01$ were excluded from analysis to remove small negative $\kappa$ values.

The relationship between $\alpha$ and $\beta$ was analyzed using regression analysis; in the analysis, $\beta$ and $\alpha$ were arbitrarily treated as independent and dependent variables respectively. Average values of $\alpha$ and $\beta$ over each combination of $Q, Y$, and $\mu$ were used in the regression analysis. The effects of $Q, Y$, and $\mu$ on the $\alpha-\beta$ relation were also investigated using regression analysis. Both $\mu$ and $Y$ were regarded as factors (categorical variables), while $Q$ (as $Q_{S}$ ) was treated as a continuous variable.

A factorial analysis of variance (ANOVA) was used to assess the overall effects of $Q_{S}, Q_{P}, Y_{0}, Y_{S}$, and $\mu$ on the five parameters $\left(\alpha, \beta, \alpha_{1}, \beta_{1}, \beta_{2}\right)$. In ANOVA, $Y_{0}$ and $Y_{S}$ were combined into a single factor, termed initial epidemic condition $(Y)$, because the combinations of $Y_{0}$ and $Y_{S}$ do not form a complete factorial set, and the emphasis of this study is on quadrat size and shape. Furthermore, in ANOVA, $Q_{S}$ was nested within each $Y$ and $\mu$ combination, and $Q_{P}$ was nested within $Q_{S}$. Therefore, the percentage of the total variance in the parameters that was accounted for by $Y$ and $\mu$ was calculated in relation to the variation between simulation runs. In contrast, the percent variance accounted for by $Q_{S}$ and $Q_{P}$ was calculated in relation to the variation within simulation runs and, therefore, cannot be

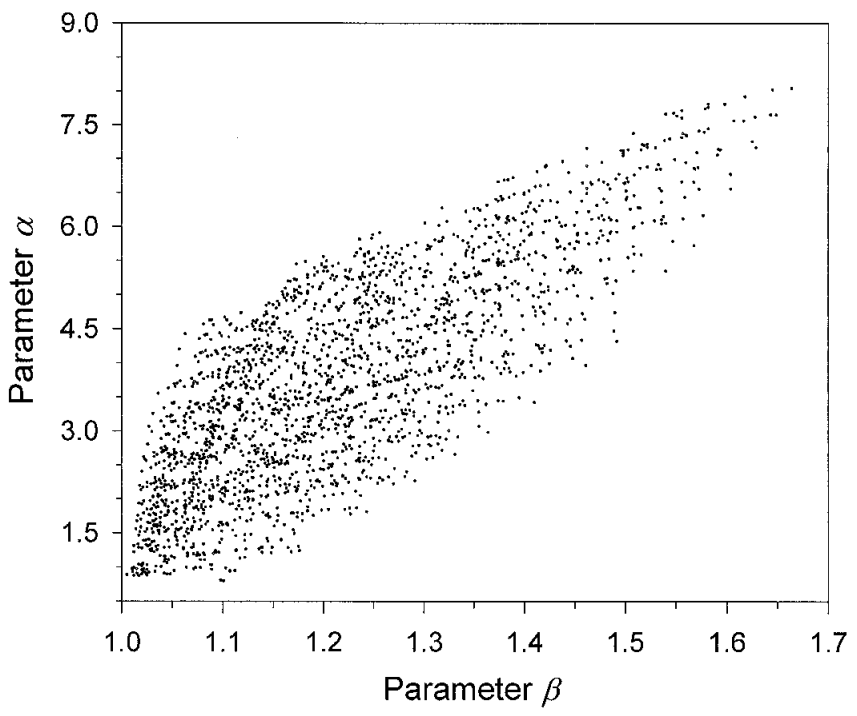

Fig. 1. Relationship of $\alpha$, averaged for each combination of $Q$ (quadrat size and shape), $Y$ (initial epidemic condition), and $\mu$ (median spore dispersal distance), with the corresponding average $\beta ; \alpha$ and $\beta$ are the two parameters of the binary power law model fitted to the simulated epidemics.

compared directly with those by $Y$ and $\mu$. To assess the effect of $Q_{S}$ on the parameters without the confounding effects of $Q_{P}$, ANOVA was also applied to square quadrats only. To assess how the effects of $\mu, Q_{S}$, and $Q_{P}$ on the parameters varied with $Y$, a separate ANOVA was performed for each of the eight initial conditions.

Regression analysis was also used to determine the relationship of the parameters with the simulation variables. In the regression analysis, both $\mu$ and $Y$ were regarded as factors (categorical variables), while $Q_{S}$ was treated as a continuous variable. All the statistical analyses were done using Genstat (Numerical Algorithms Group Ltd., Oxford).

\section{RESULTS}

Binary form of the power law. General results. The higher the value of $\mu$, the greater the rate of increase in disease incidence, as shown in the previous study (33). None of the 2,000 simulated epidemics died out (i.e., failed to increase), and equation 5, thus, was fitted to data from all 2,000 simulation runs, with a separate regression for each of the 54 quadrat types. In total, there were 108,000 regression analyses. The percent variance accounted for by equation 5 ranged from 84 to $100 \%$, with an average of $98.5 \%$. The estimated values of $\alpha$ ranged from 0.6 to 10.1 with a mean of 3.7; most values were in the range of 1 to 6 ; the $\beta$ estimates ranged from 0.93 to 1.94 , with a mean of 1.22 . The distribution of a third binary power law parameter, $\gamma=\alpha-\beta \ln (n)$, ranged from -1.88 to 0.59 , with a mean of -0.41 .

Relationship between $\alpha$ and $\beta$. There was a highly significant positive correlation between $\alpha$ and $\beta(r=0.79)$ (Fig. 1). Regression analysis showed that $\approx 63 \%$ of the total variation in $\beta$ was accounted for by $\alpha$. Including $Y$ as a factor in the model increased the variation accounted for to $81 \%$, including $\mu$ as a factor increased this further to $91 \%$ and including the logarithm of quadrat size as a covariate increased the percent variance accounted for to $96 \%$. Neither $Y$ nor $\mu$ had any significant effect on the regression coefficient of either $\alpha$ or $\ln \left(Q_{S}\right)$; furthermore, there were no significant interactions between $Y$ and $\mu$. Increasing $\mu$ and $Y_{0}$ both led to greater intercepts. The regular pattern of initial infected plants led to greater intercepts than the random pattern; in turn, the random pattern had greater intercepts than the

\begin{tabular}{|c|c|}
\hline Variable $^{c}$ & Estimate \\
\hline $\begin{array}{l}\left.\text { Constant (i.e., } Y_{1}: Y_{S}=\text { regular or clumped; } Y_{0}=1\right) \\
\alpha \\
\ln (n) \\
Y_{2}, Y_{S}=\text { random, } Y_{0}=1 \\
Y_{3}, Y_{S}=\text { clumped, } Y_{0}=4 \\
Y_{4}, Y_{S}=\text { regular, } Y_{0}=4 \\
Y_{5}, Y_{S}=\text { random, } Y_{0}=4 \\
Y_{6}, Y_{S}=\text { clumped, } Y_{0}=9 \\
Y_{7}, Y_{S}=\text { regular, } Y_{0}=9 \\
Y_{8}, Y_{S}=\text { random, } Y_{0}=9 \\
\mu=0.50^{\mathrm{d}} \\
\mu=1.0 \\
\mu=2.0 \\
\mu=4.0\end{array}$ & $\begin{aligned} & 1.086 \pm 0.0037 \\
& 0.142 \pm 0.0015 \\
&-0.130 \pm 0.0023 \\
&-0.015 \pm 0.0019 \\
& \text { As } Y_{2} \\
& 0.038 \pm 0.0023 \\
& 0.023 \pm 0.0026 \\
& \text { As } Y_{2} \\
& 0.089 \pm 0.0023 \\
& 0.054 \pm 0.0028 \\
& 0.004 \pm 0.0018 \\
& 0.014 \pm 0.0018 \\
& 0.035 \pm 0.0019 \\
& 0.078 \pm 0.0020\end{aligned}$ \\
\hline \multicolumn{2}{|c|}{$\begin{array}{l}\text { a } \alpha \text { and } \beta \text { are the two parameters of the binary power law model fitted to } \\
\text { the simulated epidemics. Model parameters are described in text } \\
\text { (equations } 1 \text { and } 5 \text { ) } \\
\text { b Initial epidemic condition }(Y) \text { and median spore dispersal distance }(\mu) \\
\text { were treated as factors and only affected the intercept (constant). } Y \text { has } \\
\text { eight levels and } \mu \text { has five levels. } Y=\text { initial conditions specified by the } \\
\text { initial number of infected plants }\left(Y_{0}=1,4 \text {, or } 9 \text { ) and their spatial pattern }\right. \\
\text { ( } Y_{S}=\text { regular, random, or clumped); } \mu=\text { median spore dispersal distance. } \\
\text { c } n=\text { sampling unit (quadrat) size } \\
\text { d } \text { For } \mu=0.25 \text {, it is zero. }\end{array}$} \\
\hline
\end{tabular}
clumped pattern (Table 1).

TABLE 1. Parameter estimates of the regression model of $\beta$ on $\alpha^{\mathrm{a}, \mathrm{b}}$ 
Parameter $\alpha$. Figure 2 shows the effects of $Y, \mu$, and $Q_{S}$ on the parameter $\alpha$, averaged over $Q_{P}$. ANOVA (Table 2) showed that $Y$ and $\mu$ accounted jointly for $75 \%$ of the total variation in $\alpha$ between simulation runs, of which $10 \%$ was due to $\mu$ alone and $4 \%$ to their interaction. The mean values of $\alpha$ with $Y_{0}=1,4$, and 9 plants were, respectively, 3.65, 3.70, and 3.82, and 3.26, 3.60, and
4.21 for the clumped, regular, and random patterns, respectively. The mean values of $\alpha$ increased from 3.57 to 4.06 as $\mu$ increased from 0.25 to 4.0 ; effects of $\mu$ decreased as the number of initial infected plants increased (Fig. 2). $Q_{S}$ accounted for $\approx 94 \%$ of the total variation in $\alpha$ within simulation runs. Increasing quadrat size led to higher $\alpha$ (Fig. 2); the rate of increase was greatest for the
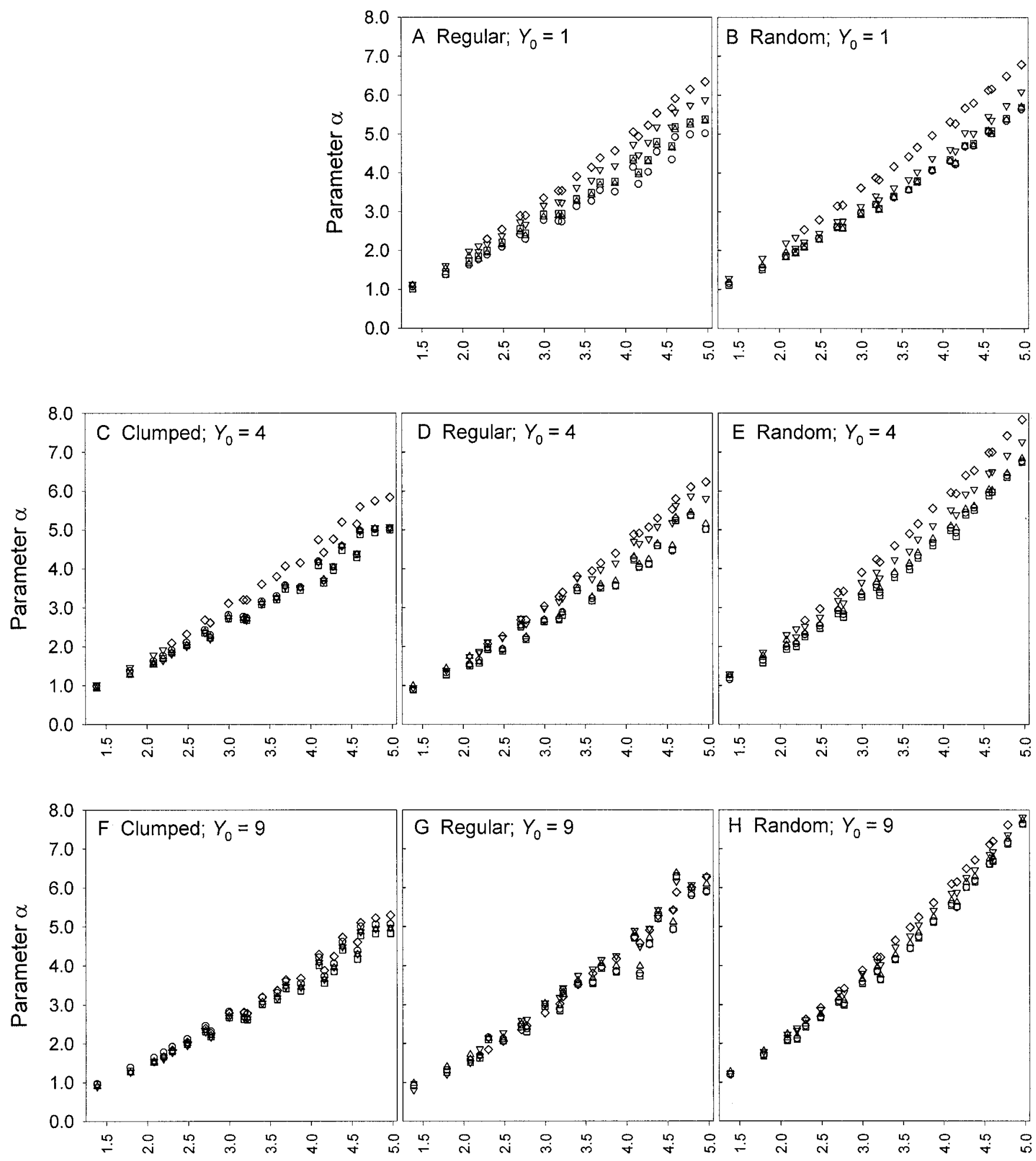

Natural logarithm of quadrat size

Fig. 2. Relationship of $\alpha$ (averaged over quadrat shapes) to the logarithm of quadrat size for each of the eight initial conditions. $\bigcirc, \square, \triangle, \nabla$, and $\diamond$ represent median spore dispersal distance $\mu=0.25,0.50,1.0,2.0$, and 4.0 , respectively. $Y_{0}=$ initial number of infected plants. 
random pattern when $Y_{0}>1$. The average values of $\alpha$ ranged from 1.06 for $Q_{S}=4$ to 6.02 for $Q_{S}=144$. The overall effects of $Q_{\mathrm{P}}$ were very small compared with $Q_{S}$ (Fig. 3A). To analyze the effects of $Q_{S}$ on $\alpha$ without the confounding effect of $Q_{P}$, a separate ANOVA was applied to the square quadrats only; the results were similar to those shown in Table 2 except that the effect of $\mu$ and $Q_{S}$ increased slightly.

To focus on how the effects of $Q_{S}$ and $Q_{P}$ on $\alpha$ varied with the initial conditions, a separate ANOVA was done for each set of initial conditions (Table 3). The contribution of $\mu$ to variation in $\alpha$ between simulation runs ranged from 11 to $49 \%$ and was greater when $Y_{0}=1$ or 4 , due mainly to the difference between $\mu=4.0$ and the others (Fig. 2). The percent variance within simulation runs due to $Q_{S}$ exceeded $95 \%$ for all eight initial conditions. The contributions of $Q_{P}$ and interactions between $Q_{S}$ and $\mu$ were small. The results of ANOVA applied only to the square quadrats were similar to those shown in Table 3.

A separate ANOVA was done for each combination of $Y$ and $Q_{S}$ to determine how the effects of $\mu$ and $Q_{P}$ on $\alpha$ varied with quadrat size within each initial condition. The percent variance accounted for by $\mu$ and $Q_{P}$ varied greatly with quadrat size. For $\mu$, it ranged from 2 to $84 \%$; for $Q_{P}$, it varied from 7 to $96 \%$. The contribution of $\mu$ to the variation in $\alpha$ generally decreased with increasing $Q_{\mathrm{S}}$, and was least for the clumped pattern. Greatest variation in $\alpha$ was attributed to $Q_{P}$ for the clumped pattern.

A regression model was used to describe the effects of quadrat size on average $\alpha$ for each combination of simulation variables (Table 4); this model was fitted to the full data set and to a restricted data set comprising only the square quadrats. For both data sets, the models accounted for more than $94 \%$ of the total variation in $\alpha$. Slope parameter $b_{1}$ depended on initial epidemic conditions, but intercept parameter $a_{1}$ did not. The value of $b_{1}$ was greatest for the random pattern. Including $\mu$ as an additional explanatory variable improved the fit of the models significantly, but the increase in the percent variance accounted for was less than $2 \%$.

Parameter $\beta$. The overall effects of $Y, \mu$, and $Q_{S}$ on parameter $\beta$, averaged over $Q_{P}$, were very similar to those on parameter $\alpha$, except that the effect of $\mu$ was considerably greater. ANOVA (Table 2) showed that $Y$ and $\mu$ accounted jointly for $82 \%$ of the total variation in $\beta$ between simulation runs; 56 and $23 \%$ were due to the main effects of $Y$ and $\mu$ respectively. The mean values of $\beta$ with $Y_{0}=1,4$, and 9 plants were, respectively, 1.17, 1.21, and 1.25 and $1.12,1.22$, and 1.28 for the clumped, regular, and random patterns, respectively. As $\mu$ increased from 0.25 to 4.0 , the mean value of $\beta$ increased from 1.17 to 1.31 , and the increase was mainly restricted to $\mu=0.5$ to 4.0 ; the effect of $\mu$ decreased with increasing $Y_{0} . Q_{S}$ accounted for $\approx 63 \%$ of total variation in $\beta$ within simulation treatments. Increasing quadrat size led to higher $\beta$; the average values of $\beta$ ranged from 1.07 for $Q_{S}=4$ to 1.34 for $Q_{S}=100$. Approximately $8 \%$ of the total variation within each simulation run was due to $Q_{P}$. Generally, the effects of $Q_{P}$ were mainly restricted to those quadrat sizes containing quadrat types with a long shape, such as $M \times 20$ or 24 (Fig. 3B). There were also large interactions between $Y$ and $Q_{S}$, accounting for approximately $12 \%$ of the total variation within simulations. The rate of the increase in $\beta$ with increasing $Q_{S}$ was least for the clumped pattern and greatest for the random pattern. ANOVA, performed only for the square quadrats, gave results very similar to those shown in Table 2.

To focus on how the effects of $\mu, Q_{S}$, and $Q_{P}$ on $\beta$ varied with initial conditions, ANOVA was applied to each $Y$ (Table 3 ). The contribution of $\mu$ to variation in $\beta$ between simulation runs ranged from 29 to $73 \%$ and was least for the clumped pattern. The percent variance due to $Q_{S}$, ranging from 54 to $87 \%$, increased with increasing $Y_{0}$, was least for the clumped pattern when $Y_{0}>1$, and was similar for the random and regular patterns. The contribution of $Q_{P}$ ranged from 7 to $39 \%$ and was greatest for the clumped pattern. Results of ANOVA applied to the square quadrats were similar to those shown in Table 3, except that the contribution of $Q_{S}$ increased considerably.

The contribution of $\mu$ and $Q_{P}$ to variation in $\beta$ within each combination of $Y$ and $Q_{\mathrm{S}}$ varied greatly with $Q_{S}$. For $\mu$, the percent variance accounted for ranged from 5 to $59 \%$; for $Q_{P}$, it ranged from 0 to $95 \%$. The interaction between $\mu$ and $Q_{P}$ was generally negligible. The contribution of $\mu$ decreased with increasing $Y_{0}$ and was less for the clumped and regular patterns. $Q_{P}$ accounted for less variation in the regular and random patterns.

A regression model was used to describe the effects of quadrat size on the average $\beta$ for each combination of simulation variables (Table 4). This model was fitted to the full data set and to a restricted data set comprising only the square quadrats. For both data sets, the model accounted for more than $86 \%$ of the total variation in $\beta$. Slope parameter $b_{2}$ depended on initial conditions, and intercept parameter $a_{2}$ depended on $\mu$. Slope $b_{2}$ was greatest for the random pattern and smallest for the clumped pattern and increased with increasing $Y_{0}$. For the intercept, there was no significant difference between $\mu=0.25$ and 0.5 , but $a_{2}$ increased with increasing $\mu$ from 0.5 to 4.0 .

Intraclass correlation. General results. Intraclass correlation $(\kappa)$ initially increased and then decreased with increasing disease incidence $(p)$; the relationship between $\kappa$ and $p$ became gradually more symmetrical from the clumped, to the regular, and then to the random pattern. The $\kappa$ values decreased with increasing quadrat size, and the maximum value of $\kappa$ decreased as $Y_{0}$ increased. Figure 4 shows the average $\kappa$ for each quadrat type over all levels of disease incidence and over all combinations of $Y$ and $\mu$. Clearly, average $\kappa$ decreased with increasing quadrat sizes. There were large differences between different quadrat types within each quadrat size (i.e., different quadrat shape). Most of the differences between quadrat shapes were due to the small $\kappa$ values for those quadrats with one extremely long side (20 or 24). Indeed, for these quadrats, the average $\kappa$ varied little as quadrat size increased (Fig. 4). Square quadrats had the greatest $\kappa$ values for a given quadrat size. Furthermore, for square quadrats average

TABLE 2. Percentage of variance accounted for by simulation variables $\left(\mu, Y, \mathrm{Q}_{s}, \mathrm{Q}_{P}\right)^{\mathrm{a}}$ in five model parameters $\left(\alpha, \beta, \alpha_{1}, \beta_{1}, \beta_{2}\right)^{\mathrm{b}}$ describing the binary power law relationship and the relationship of intraclass correlation to disease incidence

\begin{tabular}{|c|c|c|c|c|c|c|c|c|}
\hline \multirow[b]{2}{*}{ Parameter } & \multicolumn{3}{|c|}{ Between simulation runs } & \multicolumn{5}{|c|}{ Within simulation runs } \\
\hline & $\mu$ & $Y$ & $\mu \times Y$ & $Q_{S}$ & $Q_{\underline{P}}$ & $\mu \times Q_{S}$ & $Y \times Q_{S}$ & $\mu \times Y \times Q_{S}$ \\
\hline \multicolumn{9}{|c|}{ Binary power law } \\
\hline$\alpha$ & 10.3 & 60.5 & 4.1 & 94.2 & 0.5 & 0.3 & 2.3 & 0.1 \\
\hline \multicolumn{9}{|c|}{ Intraclass correlation } \\
\hline$\alpha_{1}$ & 3.9 & 49.6 & 11.4 & 6.2 & 9.7 & 0.4 & 17.2 & 1.4 \\
\hline$\beta_{1}$ & 26.8 & 51.2 & 2.6 & 52.1 & 17.6 & 0.9 & 8.4 & 0.8 \\
\hline
\end{tabular}

${ }^{a} Y=$ initial conditions specified by initial number of infected plants $\left(Y_{0}=1,4\right.$, or 9) and their spatial pattern $\left(Y_{S}=\right.$ regular, random, or clumped);

$\mu=$ median spore dispersal distance; $\mathrm{Q}_{S}=$ sampling unit (quadrat) size; $\mathrm{Q}_{P}=$ sampling unit (quadrat) shape.

${ }^{\mathrm{b}}$ Model parameters are described in the text (equations 1,5,6, and 7). 

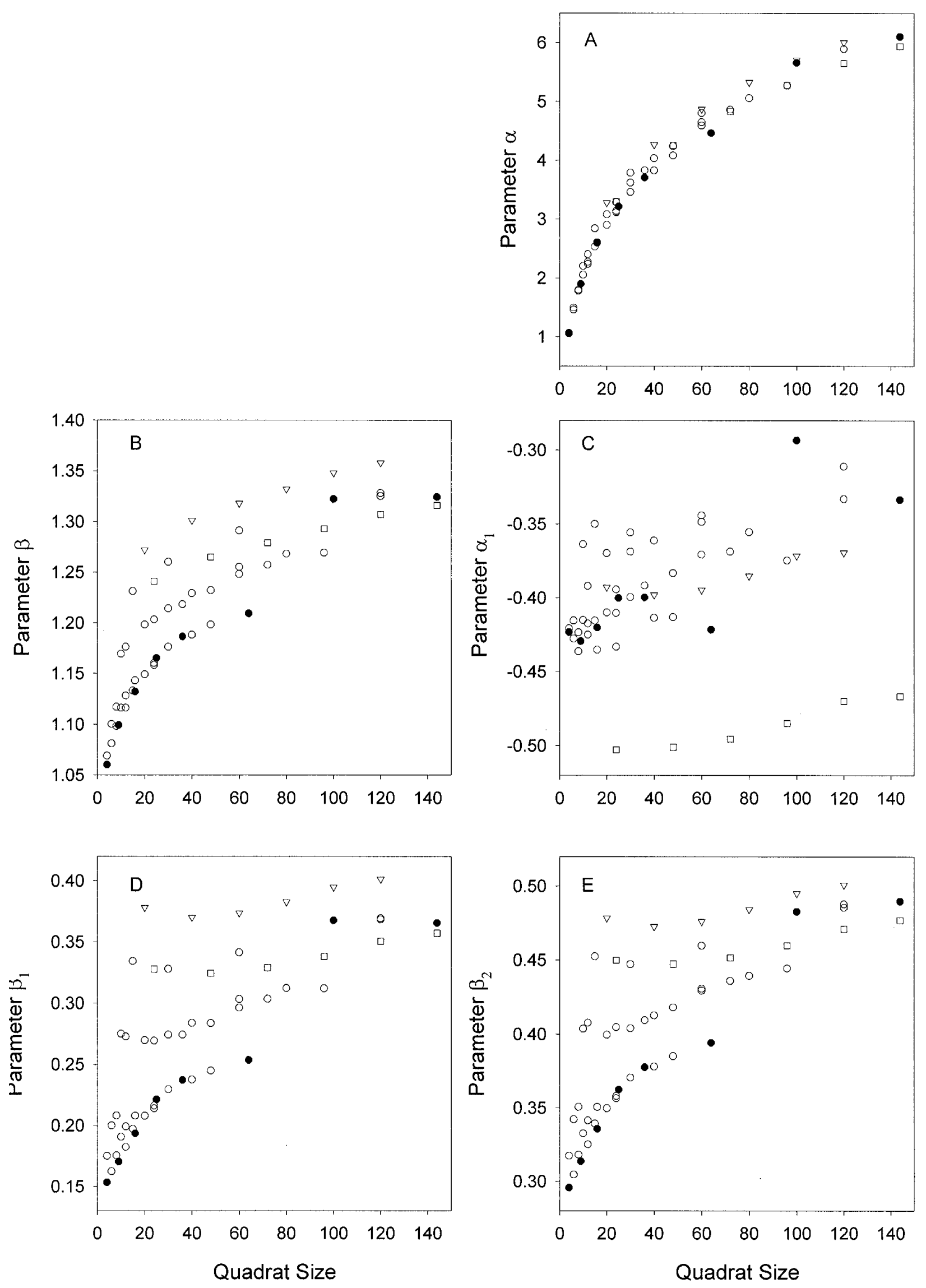

Fig. 3. The averages of parameters $\alpha, \beta, \alpha_{1}, \beta_{1}$, and $\beta_{2}$ for each of the 54 different quadrat sizes and shapes over all other simulation variables (initial conditions and spore dispersal gradient) and over all levels of disease incidence. - represents square quadrats; $\nabla$ and $\square$ represent quadrats with one side of 20 and 24 , respectively; $O$ represents all other quadrats. $\alpha_{1}, \beta_{1}$, and $\beta_{2}$ are the three parameters of the asymmetric form of the binary power law model describing the relationship of intraclass correlation to disease incidence for each simulated epidemic. 
$\kappa$ values decreased approximately exponentially with increasing quadrat size.

Equation 7 was fitted successfully to most of the 108,000 simulation runs. The percentage of variance accounted for ranged from 1 to $100 \%$, with an average of $93 \%$. There were 203 runs where the percentage of variance accounted for was less than $50 \%$, and these were excluded from the subsequent analysis. The number of epidemics with poor fit was mainly restricted to the clumped pattern (163 cases) or to $\mu=0.25$ (109 cases) or 0.5 (94 cases) but was not related to $Q_{s}$. The $\alpha_{1}$ estimated values ranged from -1.86 to 0.63 , with a mean of -0.40 . The $\beta_{1}$ estimates ranged from -0.01 to 0.89 with a mean of 0.28 . The $\beta_{2}$ estimates were more symmetrically distributed than $\beta_{1}$ and ranged from -0.14 to 1.36 , with a mean of 0.41 . All the pairwise correlations between the three parameters were small $(<0.44)$.

Parameter $\alpha_{1}$. Figure 5 shows the effects of $Y, \mu$, and $Q_{S}$ on parameter $\alpha_{1}$, averaged over $Q_{P}$. ANOVA (Table 2) showed that $Y$ and $\mu$ accounted jointly for $65 \%$ of the total variation in $\alpha_{1}$ between simulation runs, of which $4 \%$ was due to $\mu$ alone and $11 \%$ to their interaction. The mean values of $\alpha_{1}$ with $Y_{0}=1,4$, and 9 plants were, respectively, $-0.29,-0.39$, and -0.49 , and were $-0.40,-0.56$, and -0.24 for the clumped, regular, and random patterns, respectively. The mean values of $\alpha_{1}$ were similar, approximately -0.38 , as $\mu$ increased from 0.25 to 2 , but the mean value was -0.49 for $\mu=4$; the difference was mainly in simulations with initial conditions of the regular pattern at $Y_{0}=4$ or 9 and the random pattern at $Y_{0}=9$. $Q_{S}$ only accounted for approximately $6 \%$ of the total variation in $\alpha_{1}$ within simulation runs. However, there were considerable interactions between $Q_{S}$ and $Y$, accounting for approximately $17 \%$ of total variation; depending on initial conditions, increasing quadrat size could lead to lower (e.g., Fig. 5B and E) or higher $\alpha$ (Fig. 5G). $Q_{P}$ accounted for approximately $10 \%$ of the total variation in $\alpha$ within simulation runs; much of this was due to the extremely small $\alpha$ values for quadrats with one side of 24 (Fig. 3C).

A separate ANOVA was done for each set of initial conditions to determine how the effects of $Q_{S}$ and $Q_{\mathrm{P}}$ on $\alpha_{1}$ varied with initial conditions (Table 3). The contribution of $\mu$ to variation in $\alpha_{1}$ between simulation runs ranged from 4 to $78 \%$ and was much greater for the regular pattern when $Y_{0}=4$ or 9 . The percent variance within simulation runs due to $Q_{S}$ was generally small, ranging from 18 to $33 \%$. The contributions of $Q_{P}$ varied greatly from 6 to $66 \%$; it was greater for the clumped and regular patterns than for the random pattern. The interactions between $Q_{S}$ and $\mu$ were very small.

A separate ANOVA was done for each combination of $Y$ and $Q_{S}$ to determine how the effects of $\mu$ and $Q_{P}$ on $\alpha_{1}$ varied with quadrat size within each initial condition. The percent variance accounted for by $\mu$ and $Q_{P}$ varied greatly with quadrat size. For $\mu$, variance ranged from 1 to $84 \%$; for $Q_{P}$, it ranged from 0 to $93 \%$. The contribution of $\mu$ to the variation in $\alpha_{1}$ was greatest for the regular pattern when $Y_{0}>1$ (average 64\%, whereas for other initial conditions it was $9 \%$ ); the contribution of $\mu$ was not related to $Q_{S}$. The greatest variation in $\alpha_{1}$ was attributed to $Q_{P}$ for the clumped and regular patterns. In general, interactions between $\mu$ and $Q_{P}$ were small, ranging from 0 to $27 \%$, and were highest for the regular pattern.

Parameters $\beta_{1}$ and $\beta_{2}$. There was very high correlation between $\beta$ and $\beta_{1}(r=0.97): \beta_{1} \approx \beta-1$. Thus, the overall effects of simulation variables on $\beta_{1}$ were very similar to those on $\beta$ (Tables 2 and 3; Fig. 3B and D), and therefore are not presented. Figure 6 shows the effects of $Y, \mu$, and $Q_{S}$ on the parameter $\beta_{2}$, averaged over $Q_{\mathrm{P}}$. ANOVA (Table 2) showed that $Y$ and $\mu$ accounted jointly for only $27 \%$ of the total variation in $\beta_{2}$ between simulation runs.

TABLE 3. Percentage of variance accounted for by the simulation variables $\left(\mu, Q_{S}, Q_{P}\right)^{\mathrm{a}}$ in a separate analysis of the five model parameters $\left(\alpha, \beta, \alpha_{1}, \beta_{1}, \beta_{2}\right)^{\mathrm{b}}$ describing the binary power law relationship and intraclass correlation for each set of initial conditions ${ }^{c}$

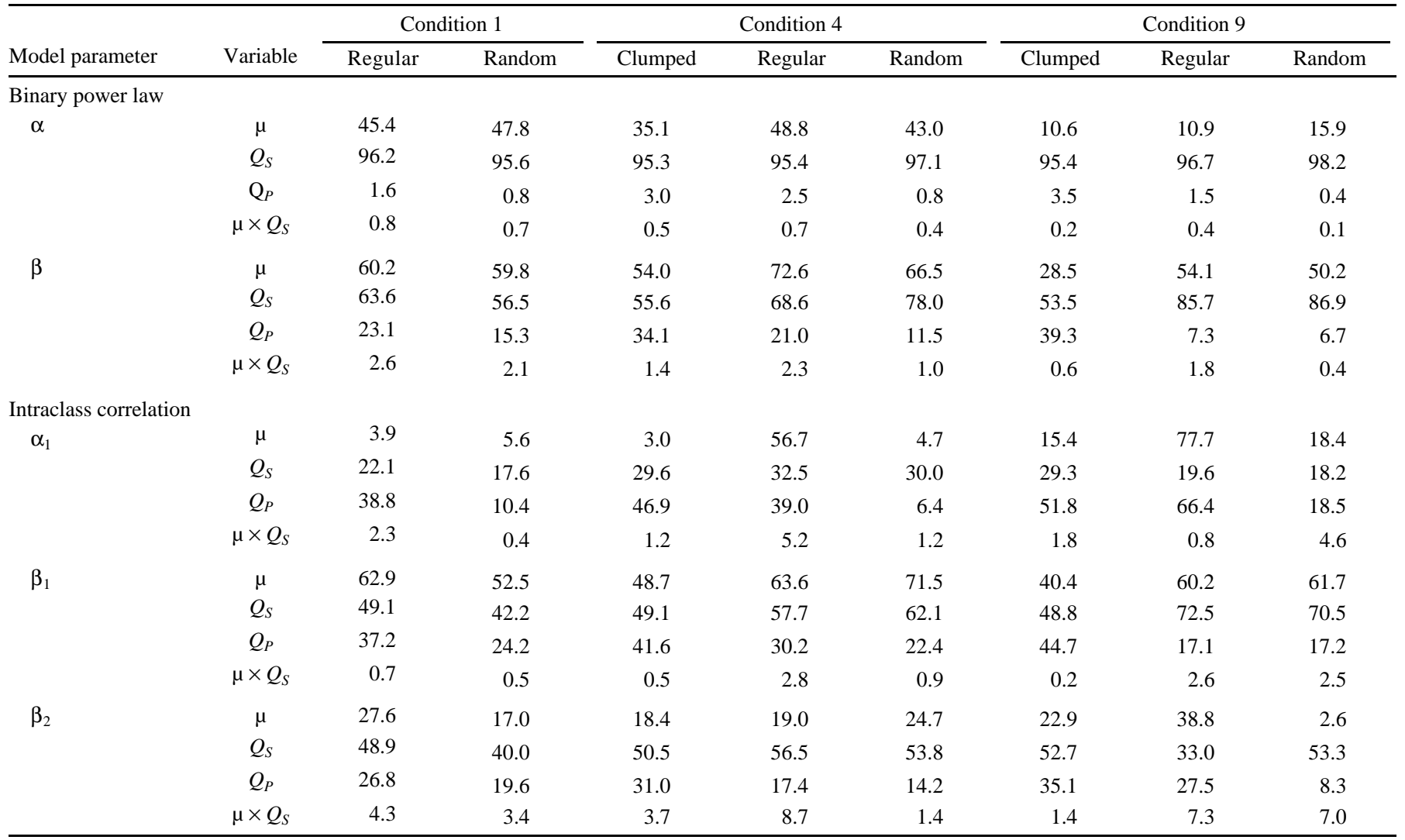

${ }^{\mathrm{a}} \mu=$ median spore dispersal distance; $\mathrm{Q}_{S}=$ sampling unit (quadrat) size; $\mathrm{Q}_{P}=$ sampling unit (quadrat) shape.

b Model parameters are described in the text (equations 1,5,6, and 7).

${ }^{c}$ Initial conditions are specified by initial number of infected plants $\left(\mathrm{Y}_{0}=1,4\right.$, or 9$)$ and their spatial pattern $\left(\mathrm{Y}_{S}=\right.$ regular , random, or clumped). 
The random pattern had the smallest $\beta_{2}$ values for $Y_{0}>1$ (Fig. 6). $Q_{S}$ accounted for approximately $46 \%$ of the total variation in $\beta_{2}$ within simulation treatments. Increasing quadrat size generally led to higher $\beta_{2}$ (Fig. 6); the average values of $\beta_{2}$ ranged from 0.31 for $Q_{S}=4$ to 0.49 for $Q_{S}=120$. Approximately $13 \%$ of the total variation within simulation runs was due to $Q_{P}$. A large proportion of the $Q_{P}$ contribution was due to the large $\beta_{2}$ values for quadrats with one side of 20 or 24 (Fig. 3E). All the interaction terms were small.

To focus on how the effects of $\mu, Q_{S}$, and $Q_{P}$ on $\beta_{2}$ varied with initial conditions, ANOVA was applied separately for each value of $Y$ (Table 3 ). The contribution of $\mu$ to variation in $\beta_{2}$ between simulation runs was generally low, ranging from 3 to $39 \%$. The contribution of $Q_{S}$ ranged from 33 to $57 \%$. The contribution of $Q_{P}$ ranged from 8 to $35 \%$ and was lowest for the random pattern. Results of ANOVA applied to the square quadrats were similar to those shown in Table 3.

The contribution of $\mu$ and $Q_{P}$ to variation in $\beta_{2}$ varied greatly between combinations of $Y$ and $Q_{S}$. For $\mu$, its contribution ranged from 2 to $97 \%$ (average 22\%); for $Q_{P}$, it ranged from 0 to $92 \%$ (average 49\%). The interaction between $\mu$ and $Q_{P}$ was generally small (average 6\%). The contribution of $\mu$ was largest for the regular pattern at $Y_{0}=9$, least for the random pattern at $Y_{0}=9$, and similar for other initial conditions. $Q_{P}$ accounted for the least and largest variation for the random and clumped patterns, respectively.

A linear regression model was used to describe the effect of the logarithm of quadrat size on average $\beta_{2}$ for each combination of simulation variables. This model was fitted to the full data set and to a restricted data set comprising only the square quadrats; for both data sets, the regression model accounted for more than $67 \%$ of the total variation in $\beta_{2}$. The slope parameter did not depend on initial conditions or $\mu$, but the intercept parameter depended on the interaction between $Y$ and $\mu$, which is why the values of these parameter estimates are not presented here. The slope estimate was greatest for the random pattern and smallest for the clumped pattern, and increased with increasing $Y_{0}$. For the intercept, there was no significant difference between $\mu=0.25$ and 0.5 but it increased with increasing $\mu$ from 0.5 to 4.0 .

Index of dispersion $(\boldsymbol{D})$. Substituting for $A(=\exp (\alpha))$ and $\beta$ in equation 3 with the equations relating $\alpha$ and $\beta$ to quadrat size and simulation variables (Table 4) gave the relationship of $D$ to disease incidence and sampling quadrat size as:

$$
D=\exp (\alpha)[(1-p) p / n]^{\beta-1}=C n^{b_{1}}[(1-p) p / n]^{a_{2}+b_{2} \ln (n)-1}
$$

where $C=\exp \left(a_{1}\right)$. Since $b_{1}$ and $b_{2}$ were dependent on initial conditions, it follows that $D$ was also influenced by initial conditions. Figure 7 shows $D$ for quadrat size up to 150 over the incidence range of 0.01 to 0.99 for three sets of initial conditions $\left(Y_{6}, Y_{7}\right.$, and $Y_{8}$ ), with parameters based on the square quadrats (Table 4). As quadrat size increased, $D$ increased with the rate of increase approximately linearly, especially for $n>30$. For the regular pattern, $D$ was much less than the random or the clumped pattern; $D$ decreased more steeply with decreasing $(p<0.5)$ or increasing $(p>0.5) p$ for the random pattern than for the clumped pattern.

\section{DISCUSSION}

The symmetrical form of the binary power law (8) successfully described all the simulated epidemics for the 54 quadrat types with size ranging from 4 to 144 plants. Estimates of the two parameters $(\alpha, \beta)$ were comparable to those estimated from real data $(16,17,19)$. As in the previous study $(33)$, the asymmetrical

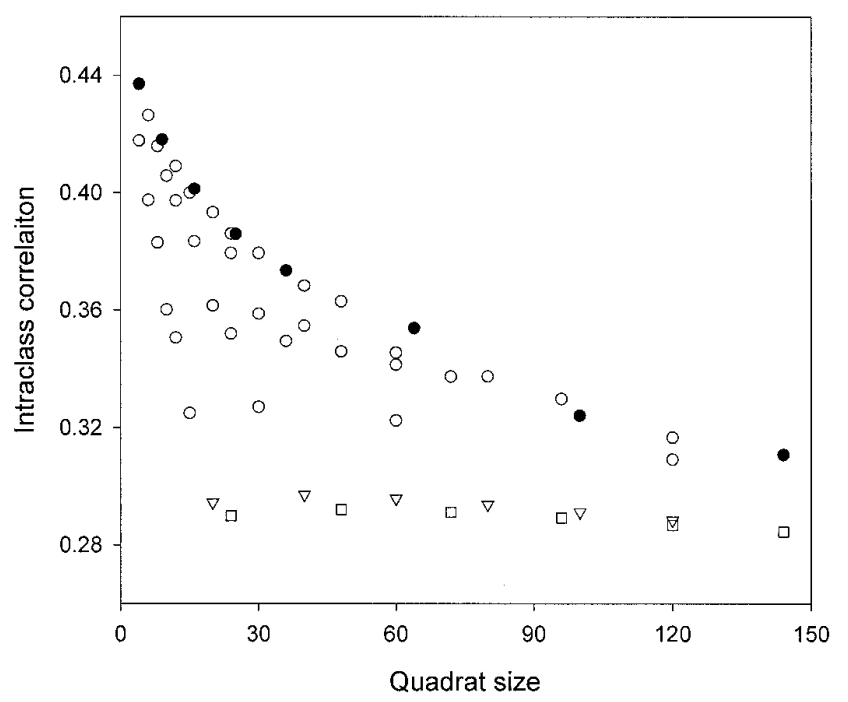

Fig. 4. Average intraclass correlation ( $\kappa)$ for each of the 54 quadrat types over all other simulation variables (initial conditions and spore dispersal gradient) and over all levels of disease incidence. represents square quadrats; $\nabla$ and $\square$ represent quadrats with one side of 20 and 24 , respectively; $\mathrm{O}$ represents all other quadrats.

TABLE 4. Parameter estimates of the models ${ }^{\mathrm{a}}$ describing the relationships of $\alpha$ and $\beta^{\mathrm{b}}$ to quadrat size and simulation variables ${ }^{\mathrm{c}}$

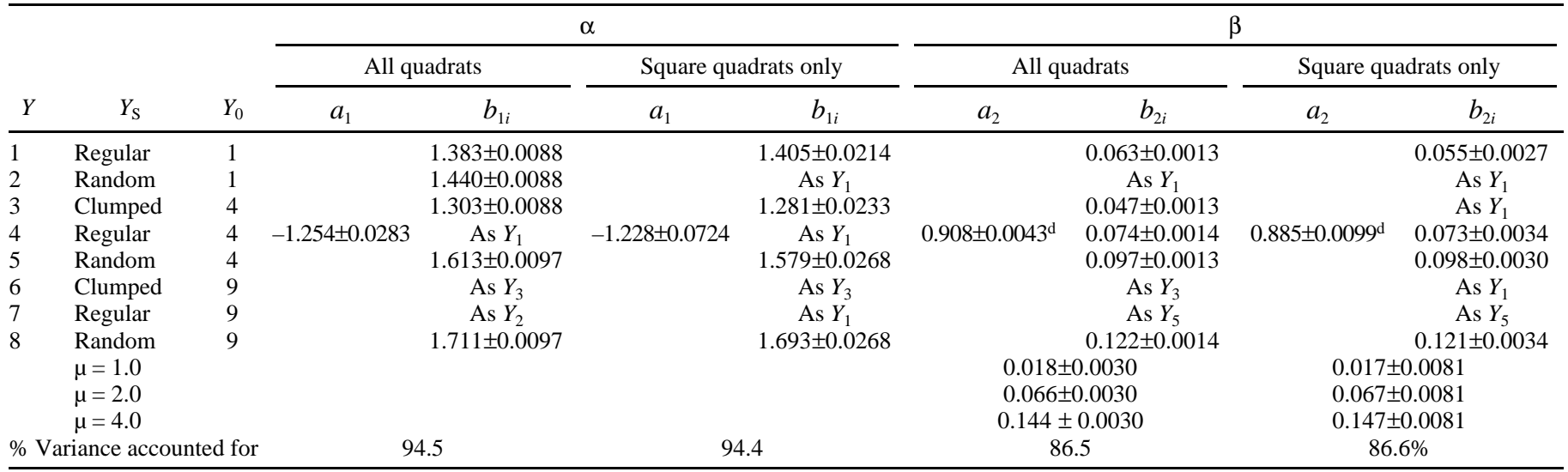

\footnotetext{
${ }^{\mathrm{a}}$ The following equations were used to model the effect of quadrat size $(n)$ on average $\alpha$ and $\beta$ for each combination of initial epidemic conditions and median spore dispersal distance: $\alpha=a_{1}+b_{1} \ln (n)$ and $\beta=a_{2}+b_{2} \ln (n)$, where $\mathrm{a}_{1}, \mathrm{~b}_{1}, \mathrm{a}_{2}$, and $\mathrm{b}_{2}$ are parameters to be estimated.

${ }^{\mathrm{b}} \alpha=$ The amplitude term in the binary power law model (16) for the variance of disease incidence data between quadrats; $\beta=$ the power term in the binary power law model.

c Simulation variables are initial conditions $(Y)$ and median spore dispersal distance $(\mu) . Y$ is specified by the initial number of infected plants $\left(Y_{0}=1,4\right.$, or 9$)$

and their spatial pattern $\left(Y_{S}=\right.$ regular, random, or clumped $)$.

d For $\mu=0.25$ or $\mu=0.5$, it is zero.
} 
form of the binary power law satisfactorily summarized the relationship between intraclass correlation and disease incidence. Analyses showed clearly that quadrat size has a large effect on the parameters of the binary power law models describing the variance-mean and intraclass correlation-incidence relationships. Furthermore, the effect of quadrat size depended on initial epidemic conditions. Compared with quadrat size, quadrat shape had small effects on the parameters.
Parameters $\alpha$ and $\beta$ were significantly positively correlated; this significant correlation could also be observed from those $\alpha$ and $\beta$ estimates obtained in experimental studies for tobacco virus diseases (8), strawberry leaf blight (30), and grape downy mildew (17). The initial epidemic conditions (number of initially infected plants and their spatial pattern) and the spore dispersal gradient $(\mu)$ had significant effects on the $\alpha-\beta$ linear relationship. For a given $\alpha$ value, increasing $\mu$ led to a greater $\beta$ value, which indicates less
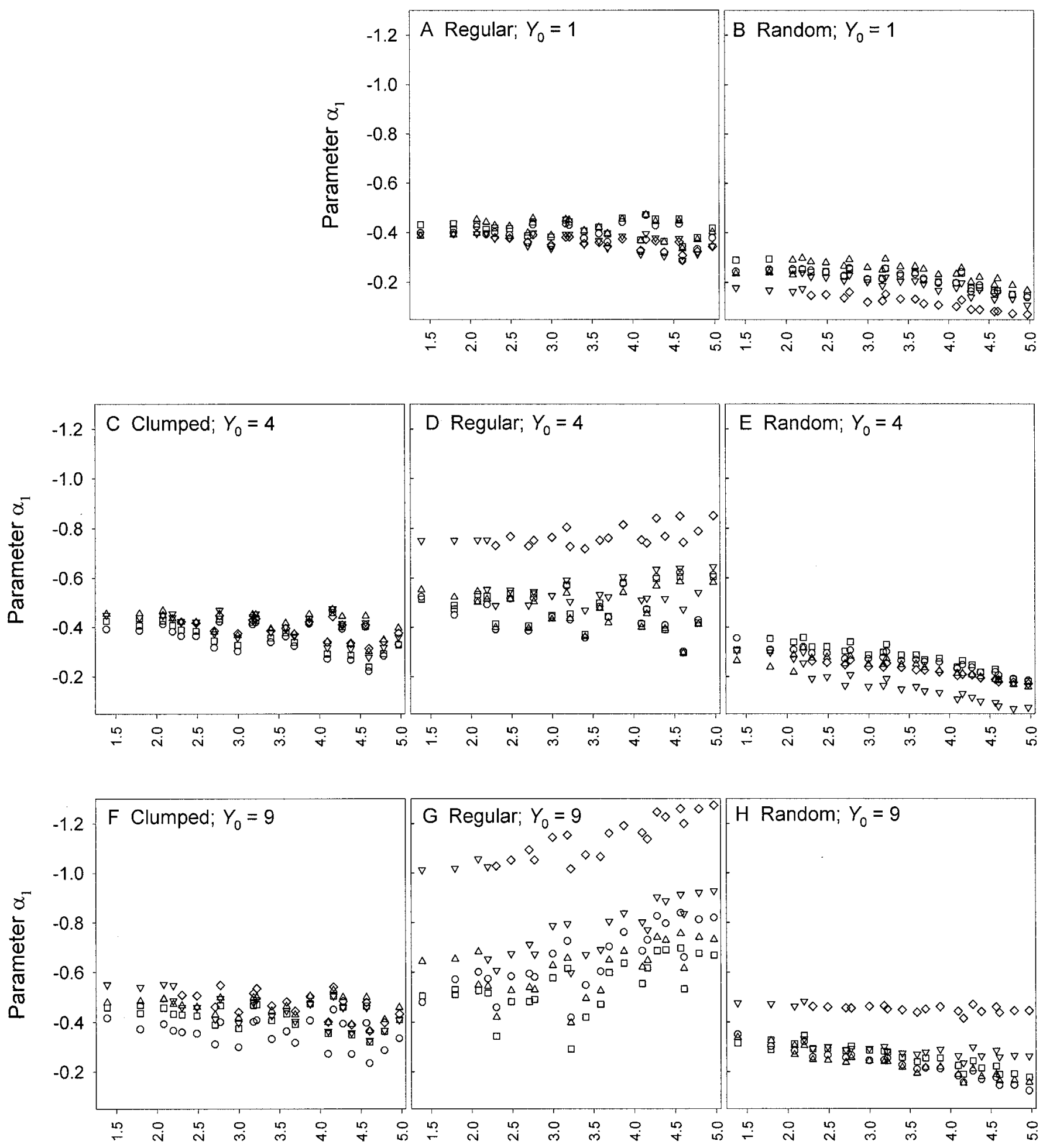

Natural logarithm of quadrat size

Fig. 5. Relationship of $\alpha_{1}$ (averaged over quadrat shapes) to the logarithm of quadrat size for each of the eight initial conditions. $\bigcirc, \square, \Delta, \nabla$ and $\diamond$ represent median spore dispersal distance $\mu=0.25,0.50,1.0,2.0$, and 4.0, respectively. $Y_{0}=$ initial number of infected plants. 
aggregation of diseased plants. Increasing $\mu$ results in more longdistance dispersal and hence is expected to result in less aggregation near the source plants. The regular and clumped patterns had the highest and lowest $\beta$ values, respectively. Increasing the number of initially infected plants led to greater $\beta$ values for the random and regular patterns. For the regular pattern, initially infected plants were least aggregated, the aggregation of initially infected plants was greatest for the clumped pattern, while aggregation of initially infected plants for the random pattern was between the two extremes. The greater the number of initially infected plants, the more likely the larger area will contain an initially infected plant for the random and regular patterns, thus leading to less aggregation over the simulation grid for these two patterns.

Initial epidemic conditions had considerable effects on the five parameters $\left(\alpha, \beta, \alpha_{1}, \beta_{1}, \beta_{2}\right)$. Furthermore, initial conditions
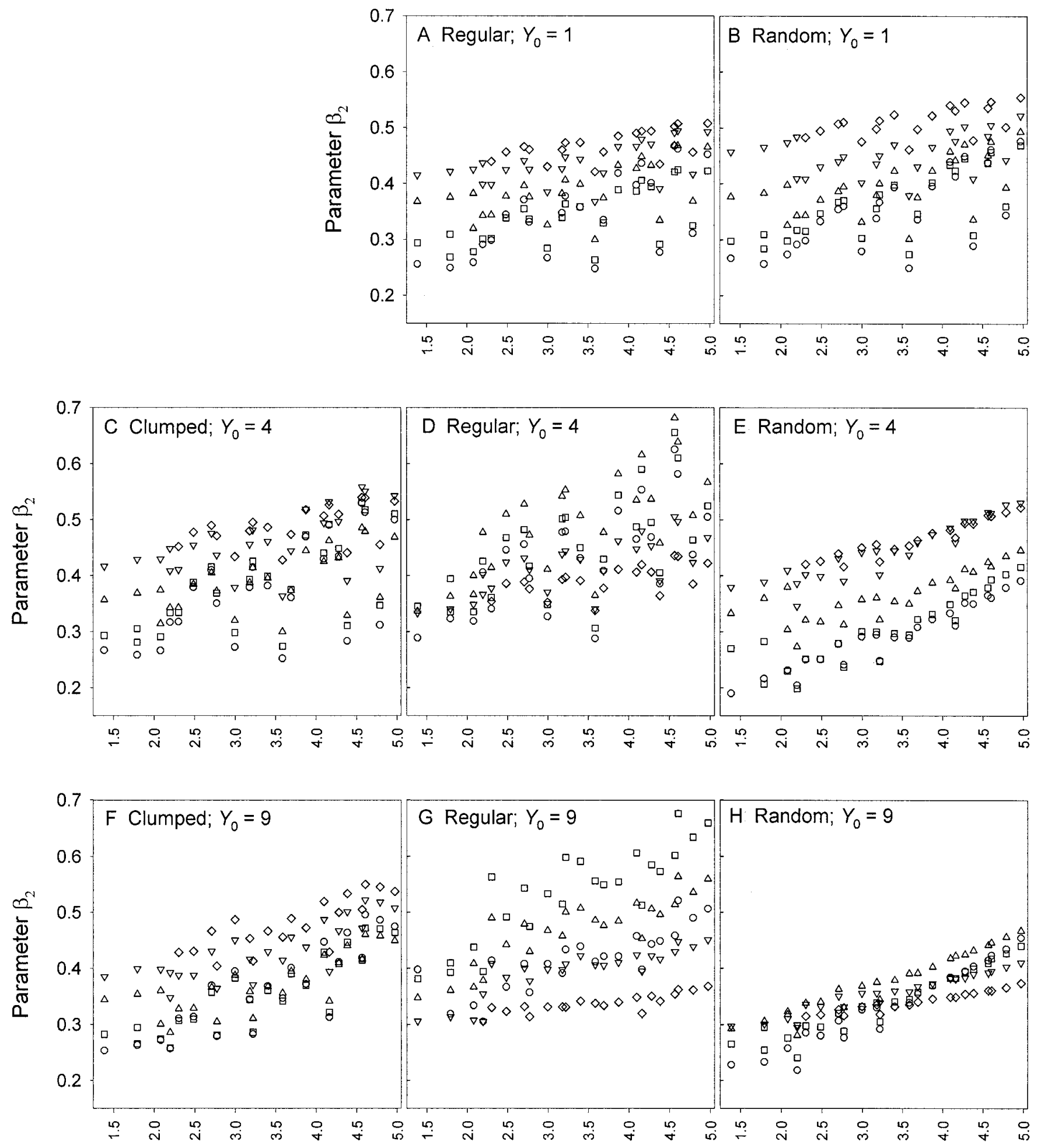

Natural logarithm of quadrat size

Fig. 6. Relationship of $\beta_{2}$ (averaged over quadrat shapes) to the logarithm of quadrat size for each of the eight initial conditions. $\bigcirc, \square, \triangle, \nabla$ and $\diamond$ represent median spore dispersal distance $\mu=0.25,0.50,1.0,2.0$, and 4.0 , respectively. $Y_{0}=$ initial number of infected plants. 


\section{A Clumped; $Y_{O}=9$}

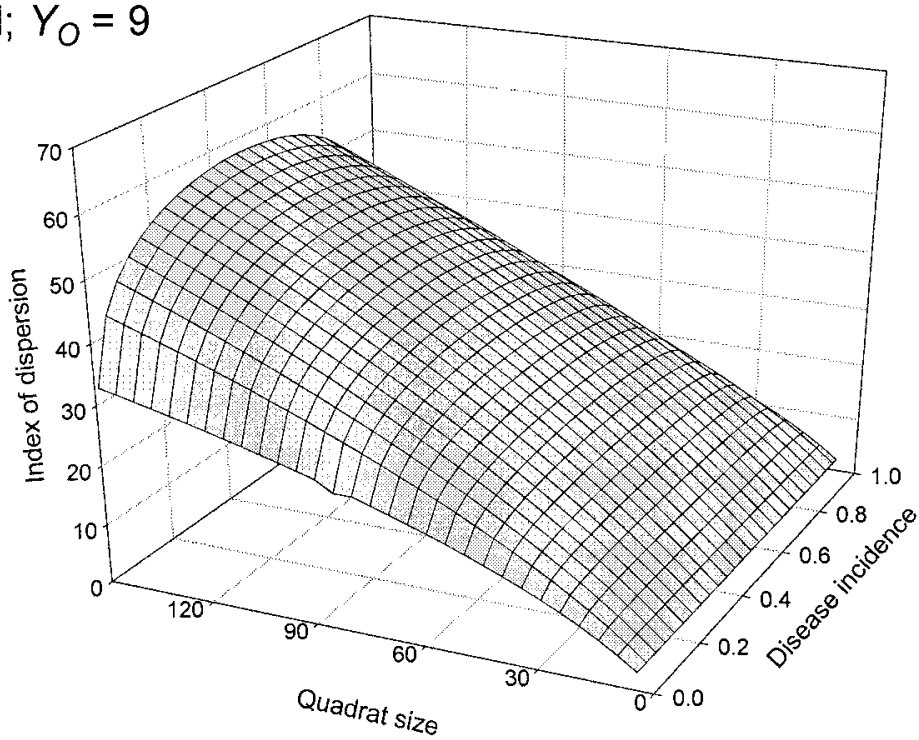

B Regular; $Y_{O}=9$

\section{Random; $Y_{O}=9$}
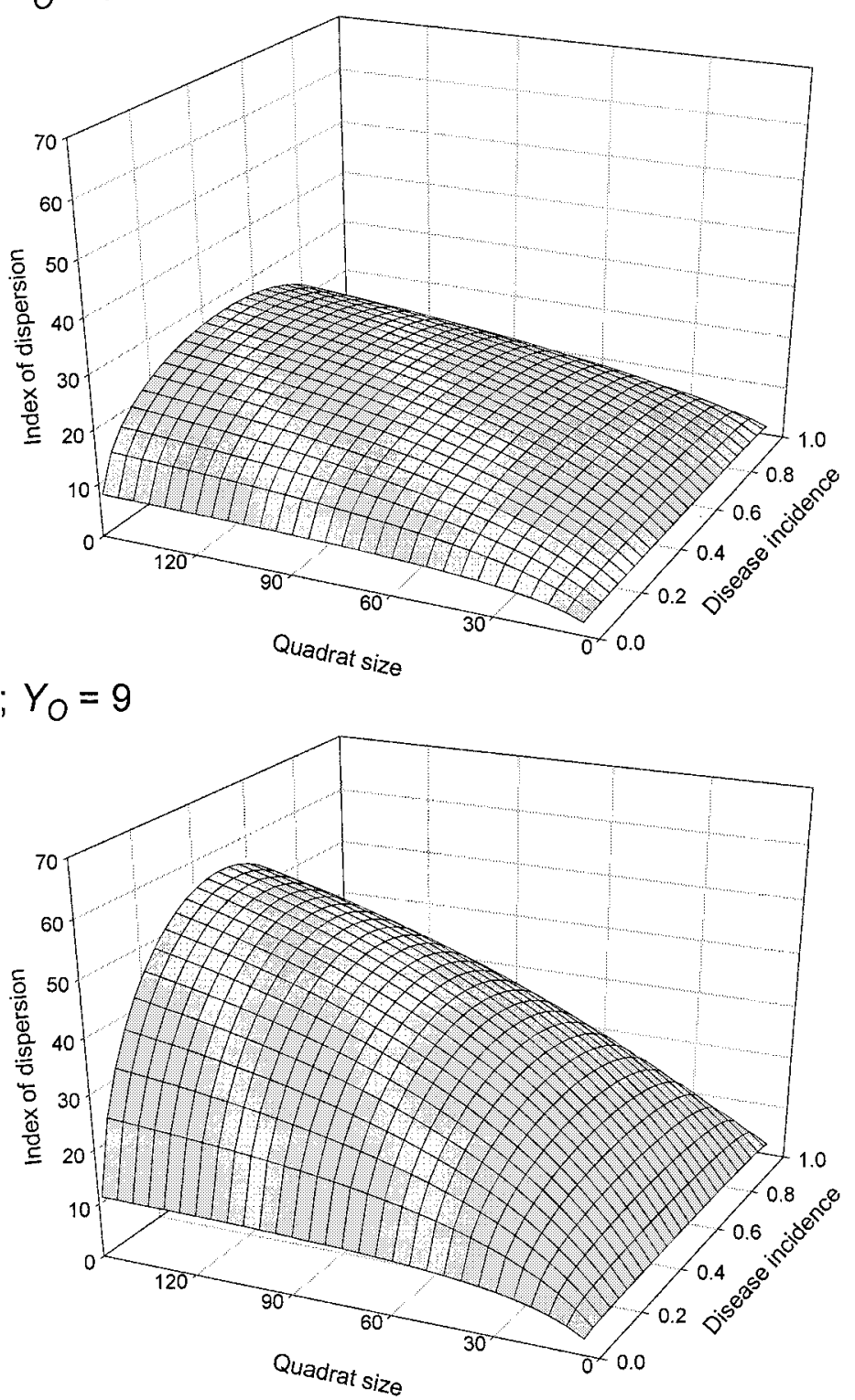

Fig. 7. The index of dispersion in relation to disease incidence and quadrat size calculated using equation 8 . The index is based on the models derived from the square quadrats only for three of the eight initial conditions (Table 4). $Y_{0}=$ initial number of infected plants. 
affected the relative contributions of quadrat size and shape and the spore dispersal gradient $(\mu)$ to variation in these parameters. The importance of initial epidemic conditions on the spatiotemporal dynamics of plant diseases has been demonstrated previously, theoretically and experimentally. In an experiment with southern blight of processing carrot, the rate of disease increase generally increased as the number of initial disease foci increased (27). An aggregated pattern of initial disease resulted in lower disease incidence than a uniform pattern for Zucchini yellow mosaic virus (22) and rice sheath blight (24). Analytical and simulation modeling studies have shown that aggregation slows the rate of disease development $(20,31,33,36)$ and that spatial statistics are affected significantly by the initial conditions $(33,35)$.

As expected, increasing $\mu$ led to less aggregation, i.e. smaller $D$ or $\kappa$ values, as it led to more long-distance dispersal. However, its overall effect on $\alpha, \beta, \alpha_{1}, \beta_{1}$, and $\beta_{2}$ was generally small compared with that of initial conditions, but it varied greatly with initial conditions, confirming previous simulation studies (33). In general, for each set of initial conditions, $\mu$ explained a greater proportion of the variation in $\alpha, \beta$ and $\beta_{1}$ than in $\alpha_{1}$ or $\beta_{2}$. Furthermore, the effects of $\mu$ on the parameters were much greater for the regular pattern than for the other patterns. This is likely to be due to the confounding effects of aggregation of initially infected plants with subsequent aggregation due to $\mu$ during the epidemic development period. For the regular pattern, initially infected plants were least aggregated when the epidemic started, thus aggregation of subsequent diseased plants due to $\mu$ was least confounded with the aggregation of initially infected plants.

Compared with other factors, quadrat size was the single most important factor affecting spatial statistics such as intraclass correlation, index of dispersion, and binary power law. An effect of quadrat size on spatiotemporal statistics was expected theoretically $(15,16,34)$ and has been demonstrated experimentally $(6,19)$. For example, heterogeneity for strawberry Phomopsis leaf blight, measured as the beta-binomial parameter $\theta$, index of dispersion, and the binary power law parameters, was affected by the number of sampling units (15 leaflets or 5 leaves) per quadrat (30). Increasing quadrat size resulted in greater values of $\alpha, \beta, \alpha_{1}$, $\beta_{1}$, and $\beta_{2}$. Indeed, more than $90 \%$ of the total variation in $\alpha$ was accounted for by a simple linear regression on the logarithm of quadrat size, with the slope parameter depending on initial conditions. Theoretically, the constant terms in these empirical models relating $\alpha$ and $\beta$ to the logarithm of quadrat size (equations 8 and 9) should be 0 and 1 respectively because, for a quadrat of size $1 \alpha$ and $\beta$ are expected to be $0(A=1)$ and 1 , respectively. However, setting the constants to zero or one as appropriate resulted in models that significantly overestimated and underestimated $\alpha$ and $\beta$ at small and large quadrat sizes, respectively, indicating some nonlinear feature in the relationship. Nonetheless, the linear relationships provide a reasonable empirical fit to the data over the range of quadrat sizes considered. The relationship between quadrat sizes and the parameters was of a log-linear type. However, it is not immediately clear why the relationship should be of this form. It might be due to the Cauchy spore dispersal distribution used in the simulation model or to the nature of quadrat sampling, or to other causes. Further studies are needed to understand this.

Intraclass correlation $(\kappa)$ increased initially and then declined with increasing disease incidence $(p)$. This relationship has been observed in several experiments $(17,19,30)$ (the beta-binomial parameter $\theta$ instead of $\kappa$ was used $[17,30]$ : $\kappa$ is a monotonic function of $\theta$ ). Increasing quadrat size led to decreasing $\kappa$ values; again this relationship is of log-linear form. Declines in intraclass correlation with quadrat size have been observed by Madden et al. (19) and Tanne et al. (28); these authors showed that the betabinomial parameter $\theta$ decreased with increasing quadrat size. It is expected that $\kappa$ will decrease as quadrat size increases. Intraclass correlation $\kappa$ is defined as the mean of the complete set of pairwise correlations between plants within a quadrat. Spatial autocorrelation of individual plants declined with distance for this simulation model (33) and in experimental studies for citrus canker (7) and strawberry leather rot (23). As the quadrat size increases, the average distance between a pair of plants within quadrats will increase and, thus, lead to smaller $\kappa$. Furthermore, the previous study (33) showed that spatial autocorrelation declines approximately exponentially with distance for this simulation model, which may explain the observed log-linear relationship between $\kappa$ and quadrat size. Increasing quadrat size led to decreasing $\kappa$ values, implying less aggregation. However, the index of dispersion $(D)$ increased with quadrat size, approximately linearly for quadrat size $>30$; this relationship is critically dependent on initial conditions. Since $D=1+\kappa(n-1)$, it implies that the rate of decline in $\kappa$ with increasing quadrat size must be less than $1 /(n-1)$. Thus, increasing quadrat sizes led to apparently contradictory results: smaller $\kappa$ indicated less aggregation, larger $D$ indicated greater aggregation. This stresses the importance of interpreting spatial statistics correctly.

Compared with the effect of quadrat size, the effect of quadrat shape was generally small, especially for $\alpha$ and $\beta$. However, there were large effects for certain sets of initial conditions and quadrat sizes. The greatest differences in spatial statistics were observed between square quadrats and quadrats of the same size but with a very long side (i.e., 20 or 24). Indeed, average intraclass correlation for these long and thin quadrats was very small and did not vary with increasing quadrat size. This may be due to the fact that, for the extremely long-shaped quadrats, $\kappa$ was predominantly determined by the pairwise correlations of plants over a larger distance, which is very small. Generally the effect of quadrat shape was smaller for the random initial pattern than for the clumped or regular patterns. Some caution may be needed in interpreting the effects of quadrat shape because of the limited range of quadrat shapes that are available within each quadrat size.

This study shows clearly that spatiotemporal statistics derived from quadrat data are influenced greatly by quadrat size and initial epidemic conditions, even though within a fixed quadrat size under a particular set of initial conditions the effects of sporedispersal gradient and quadrat shape can be large and significant. Thus, it is very important to select appropriate initial conditions in experiments for obtaining maximum information and to avoid using extreme long-shaped quadrats for sampling. Furthermore, the differences among the values of these spatial statistics reported from different studies may reflect, at least in part, differences in quadrat sizes and initial conditions and, to a lesser extent, differences in the spore-dispersal gradient.

\section{LITERATURE CITED}

1. Campbell, C. L., and Madden, L. V. 1990. Introduction to Plant Disease Epidemiology. John Wiley \& Sons, New York.

2. Cochran, W. G. 1977. Sampling Techniques. 3rd ed. John Wiley \& Sons, New York.

3. Fisher, R. A. 1925. Statistical Methods for Research Workers. Oliver and Boyd, Edinburgh.

4. Fleiss, J. L. 1981. Statistical methods for rates and proportions. 2nd ed. John Wiley \& Sons, New York.

5. Gottwald, T. R. 1995. Spatio-temporal analysis and isopath dynamics of citrus scab in nursery plots. Phytopathology 85:1082-1092.

6. Gottwald, T. R., Avinent, L., Llacer, G., Hermoso de Mendoza, A., and Cambra, M. 1995. Analysis of spatial spread of sharka (plum pox virus) in apricot and peach orchards in eastern Spain. Plant Dis. 79:266-278.

7. Gottwald, T. R., Reynolds, K. M., Campbell, C. L., and Timmer, L. W. 1992. Spatial and spatiotemporal autocorrelation analysis of citrus canker epidemics in citrus nurseries and groves in Argentina. Phytopathology 82:843-851.

8. Hughes, G., and Madden, L. V. 1992. Aggregation and incidence of disease. Plant Pathol. 41:657-660.

9. Hughes, G., and Madden, L. V. 1993. Using the beta-binomial distribution to describe aggregated patterns of disease incidence. 
Phytopathology 83:759-763.

10. Hughes, G., Madden, L. V., and Munkvold, G. P. 1996. Cluster sampling for disease incidence data. Phytopathology 86:132-137.

11. Hughes, G., McRoberts, N., Madden, L. V., and Nelson, S. C. 1997. Validating mathematical models of plant-disease progress in space and time. IMA J. Math. Appl. Med. \& Bio. 14:85-112.

12. Jeger, M. J. 1989. Spatial Components of Plant Disease Epidemics. Prentice Hall, New Jersey.

13. Kranz, J. 1974. Comparison of epidemics. Annu. Rev. Phytopathol. 12: 355-374.

14. Kranz, J. 1978. Comparative anatomy of epidemics. Pages 33-62 in: Plant Disease. Vol. 2, How Disease Develops in Populations. J. G. Horsfall, and E. B. Crowling, eds. Academic Press, New York.

15. Li, H., and Reynolds, J. F. 1995. On definition and quantification of heterogeneity. Oikos 73:280-284.

16. Madden, L. V., and Hughes, G. 1995. Plant disease incidence: Distributions, heterogeneity, and temporal analysis. Annu. Rev. Phytopathol. 33:529-564.

17. Madden, L. V., Hughes, G., and Ellis, M. A. 1995. Spatial heterogeneity of the incidence of grape downy mildew. Phytopathology 85:269-275.

18. Madden, L. V., Hughes, G., and Munkvold, G. P. 1996. Plant disease incidence: Inverse sampling, sequential sampling, and confidence intervals when observed mean incidence is zero. Crop Prot. 15:621-632.

19. Madden, L. V., Nault, L. R., Murral, D. J., and Apelt, M. R. 1995. Spatial pattern analysis of the incidence of aster yellows disease in lettuce. Res. Popul. Ecol. 37:279-289.

20. McRoberts, N., Hughes, G., and Madden, L. V. 1996. Incorporating spatial variability into simple disease progress models for crop pathogens. Pages 75-82 in: Aspects of Applied Biology. Assoc. Appl. Biologists, Wellesbourne, Warwick, England./Vol. 46, Modelling in Applied Biology: Spatial aspects.

21. Mollison, D. 1977. Spatial contact models for ecological and epidemic spread. J. Roy. Stat. Soc., Ser. B 39:283-326.

22. Nelson, S. C. 1996. A simple analysis of disease foci. Phytopathology 86:332-339.

23. Reynolds, K. M., Madden, L. V., and Ellis, M. A. 1988. Spatio-temporal analysis of epidemic development of leather rot of strawberry. Phytopathology 78:246-252.

24. Savary, S., Willocquet, L., and Teng, P. 1997. Modelling sheath blight epidemics in rice tillers. Agric. Sys. 55:359-384.

25. Shaw, M. W. 1994. Modeling stochastic processes in plant pathology. Annu. Rev. Phytopathol. 32: 523-544.

26. Shaw, M. W. 1995. Simulation of population expansion and spatial pattern when individual dispersal distributions do not decline exponentially with distance. Proc. of The R. Soc. (Lond.), Ser. B 259:243-248.

27. Smith, V. L., Campbell, C. L., Jenkins, S. F., and Benson, D. M. 1988. Effects of host density and number of disease foci on epidemics of southern blight of processing carrot. Phytopathology 78:595-600.

28. Tanne, E., Marcus, R., Dubitzky, E., and Raccah, B. 1996. Analysis of progress and spatial pattern of corky bark in grapes. Plant Dis. 80:34-38.

29. Taylor, L. R. 1961. Aggregation, variance and the mean. Nature (Lond.) 189:732-735.

30. Turechek, W. W., and Madden, L. V. 1999. Spatial pattern analysis of strawberry leaf blight in perennial production systems. Phytopathology 89: 421-433.

31. Waggoner, P. E., and Rich, S. 1981. Lesion distribution, multiple infection, and the logistic increase of plant disease. Proc. of the Natl. Acad. Sci. USA 78:3292-3295.

32. Wichmann, B. A., and Hill, I. D. 1982. An efficient and portable pseudorandom number generator. Appl. Stat. 31:188-190.

33. Xu, X.-M., and Ridout, M. S. 1998. Effects of initial epidemic conditions, sporulation rate, and spore dispersal gradient on the spatiotemporal dynamics of plant disease epidemics. Phytopathology 88:1000-1012.

34. Yamamura, K. 1990. Sampling scale dependence of Taylor's power law. Oikos 59:121-125.

35. Yang, X. B. 1995. Analysis of variance-mean relationships of plant diseases. Phytopathology 143:513-518.

36. Yang, X. B., and TeBeest, D. O. 1992. Dynamic pathogen distribution and logistic increase of plant disease. Phytopathology 82:380-383.

37. Zadoks, J. C., and van den Bosch, F. 1994. Expansion and spatial spread of disease. Annu. Rev. Phytopathol. 32:503-521. 